\title{
STUDY OF ULTRA-VIOLET RADIATION IN THE TREATMENT OF TUBERCULOSIS IN CHILDHOOD.
}

\author{
BY \\ T. HARTLEY MARTIN, M.B., СH.В., \\ and JOHN D. CRAIG, M.D. \\ (From the Open Air Hospital for Children, Leasowe.)
}

I. Introduction. (T. Hartley Martin).

II. The Sedimentation Rate and Blood Pressure as criteria of the value of Ultra-Violet Radiation in Tuberculosis of Childhood. (John D. Craig).
III. Observations on Endocrine Activities and Carbohydrate Tolerance IN CONNECTION WITH ULTRA-VIOLET Radiation. (John D. Craig).

IV. Experimental Data and Conclusions. (John D. Craig).

\section{INTRODUCTION.}

In an endeavour to explore every avenue for the treatment of nonpulmonary tuberculosis in children a visit was paid to the Finsen Institute in Copenhagen in October, 1921, to gain first hand knowledge of the methods employed at that centre in view of the results claimed.

Although between 80 and 85 per cent. of the cases treated in this hospital by fixation and natural helio-therapy were discharged quiescent, it was felt that artificial light might secure (1) a greater percentage of 'recoveries'; (2) a reduction in the time required to attain ' recovery' ; (3) an amelioration in the condition of advanced and complicated cases which had failed to respond to open-air treatment.

The visit to Copenhagen was of too short a duration to confirm with certainty the above-mentioned queries, but it was felt that sufficient evidence had been produced to allow of an experimental installation of artificial light. Two long-flame carbon arc lamps were therefore installed in August, 1922, and the following technique was used :-Routine acclimatization was commenced, 10 minutes ( 5 back, and 5 front) at 3 feet being the initial dose. This was increased by 10 minutes every other day until a full hour could be given three times a week. Departures from this routine were made in cases where idiosyncrasy to any given doses was shown.

The results achieved over six years have been encouraging. The most marked advantage obtained by artificial heliotherapy has been the ability to 
supply regular stimulus by means of organized periodic doses of light, and this has undoubtedly reflected itself upon the local foci of disease because of the marked improvement in the general condition of the children treated.

Although it is as yet too early to say that greater percentage of recoveries has been obtained, and although it has been definitely established that the cases in an advanced stage of disease do not benefit by such treatment but are rather made worse, there is definite evidence to show that the duration of stay in hospital of cases of tuberculous adenitis and tuberculous peritonitis has been lessened, and that fewer of these cases relapse on return to home life than was the case formerly. It was, however, felt that more conclusive evidence as to the value of artificial light treatment should be obtained, and, in order to attempt to secure this, research work was undertaken by Dr. John D. Craig, the details of which are amplified in the accompanying articles.

The results obtained were satisfactory up to a certain point and it is felt that the conclusions warranted a continuance of such research over a longer period and the production of further evidence as a result of experiments of (1) treatment by ' light' in the earliest stages of disease ; $(2)$ the reaction to 'light' of varying wave lengths.

\section{THE SEDIMENTATION RATE AND BLOOD PRESSURE AS CRITERIA OF THE VALUE OF ULTRA-VIOLET RADIATION IN TUBERCULOSIS OF CHILDHOOD.}

\section{The Sedimentation Rate of Erythrocytes.}

In the days when blood letting was common it was a recognised phenomenon that the settling rate of blood corpuscles with the formation of a 'cruor sanguinis' was increased in inflammatory conditions and this was considered to be an important clinical sign by Hewson (1771) and Hunter (1794).

Probably on account of the decline of blood-letting the test was allowed to lecome dormant until 1917 when Fohrœus ${ }^{1}$ drew attention to it, and again in 1921 when Westergren ${ }^{2}$ published an account of his technique and conclusions. Since that time an immense literature has appeared, most of it emanating from Continental centres, but some more recently from America.

In England Heaf ${ }^{3}$, Lyle Cummins and Acland ${ }^{4}$, and R. G. Bannerman ${ }^{5}$ have made contributions to the literature.

Most of the work referred to above has been done with a view to using the sedimentation rate as a test in diagnosis, and in this respect it has been disappointing ; for, as is shown in the following notes, its value as an aid in diagnosis is limited. In one aspect, however, namely as an aid to prognosis, and particularly as a criterion of the effect of treatment, there is almost unanimous approval that it is of value. 
It was during the period when search was being made in the literature for assistance in assessing the value of ultra-violet radiation that attention was drawn to Heaf's ${ }^{3}$ work on this test, and further enquiries supported his views. Because of this it was felt that there was justification for further experimentation with this test for the purpose in view.

Mode of action. The factors controlling the sedimentation reaction are not yet fully understood. Fohrous demonstrated that in rapidly sedimenting blood there is a relative excess of fibrinogen and globulin as against albumen, and that the auto-agglutination of red blood corpuscles is an essential element, since on physical grounds a clump of red blood corpuscles will fall at a greater speed than discreet cells. Bannerman ${ }^{5}$ states that the number of red blood corpuscles has but little influence on the sedimentation rate, which was found in a case of severe anæmia to be within normal limits. Beaumont and Dodds? suggest that breaking down of tissue proteins may account for an increased rate of sedimentation.

The suggestion is made that for the purposes of translating the month to month variation of this test the rate of fall is in proportion to the activity of the disease. Thus a rise in the rate of sedimentation, i.e., diminished numerical reading, is taken to mean a temporary increase in the activity in the focus of disease, whereas a fall in the rate, i.e., a higher numerical reading, implies a temporary decline in the activity of the disease.

Westergren gives the following figures for the classes into which observations fall :-

1. Clear fluid column of $3 \mathrm{~mm}$. (197 mm-200 mm.) is normal.

2. " " " , 4-6 mm. (194 mm.-196 mm.) is doubtful.

3. " " " , 7-12 mm. (188 mm.-193 mm.) is probably pathological.

4. " " " over $12 \mathrm{~mm}$. (under $188 \mathrm{~mm}$.) is certainly pathological.

Technique. $\quad 0.4 \mathrm{c.cm}$. of a 3.8 per cent. solution of sodium citrate in sterile distilled water is drawn up into a 2 c.cm. syringe. Slight ligature-pressure, which is necessary in children, is then applied to the upper arm and the patient's median basilic vein punctured. $1.6 \mathrm{c} . \mathrm{cm}$. of blood is withdrawn so that the syringe is now filled with blood and citrate up to the $2 \mathrm{c.cm}$. mark without any air bubbles intervening. The contents of the syringe are now placed slowly into a small dry gallipot.

A pipette holding $1.25 \mathrm{c.cm}$. graduated individually into 200 divisions is taken, and drawing up the citrated blood at least five times thoroughly mixes the contents of the gallipot. It is finally sucked up to the $200 \mathrm{~mm}$. mark on the pipette, care being taken to obtain a column of blood without air bubbles. The pipette is placed in a vertical position on a special stand and the reading of the height of the column of red blood corpuscles taken at the end of one hour.

Various investigators have taken readings at hourly intervals for 24 hours, but in this work the one-hour reading has been used as it is generally agreed that for comparative purposes it is the most useful. To verify the results of others, a series of cases were examined at the beginning in which the readings were taken at the first, second, third, fourth, fifth, sixth, ninth, twelfth, eighteenth and twenty-fourth hours. The results agreed within limits with Heaf, and thereafter the one-hour reading was the only one taken. 
TABLE I.

Sedimentation Rates and Blood Pressure Readings in Cases treated by Ultra-Violet Radiation.

\begin{tabular}{|c|c|c|c|c|c|c|c|c|c|c|c|}
\hline \multirow{2}{*}{$\begin{array}{c}\text { Case } \\
\text { No. }\end{array}$} & \multicolumn{2}{|c|}{ Diagnosis. } & \multirow{2}{*}{$\frac{\text { Stage. }}{\text { C.3. }}$} & \multicolumn{7}{|c|}{$\begin{array}{l}\text { Monthly Readings of Sedimentation Rates } \\
\text { and Blood Pressure. }\end{array}$} & \multirow{2}{*}{$\begin{array}{l}\text { Clinical Progress. } \\
\text { No change. }\end{array}$} \\
\hline & Spine & . & & $\begin{array}{l}\text { S.R. } 121 \\
\text { B.P. } 106\end{array}$ & $\begin{array}{l}135 \\
110\end{array}$ & $\begin{array}{r}125 \\
94\end{array}$ & $\begin{array}{r}116 \\
86\end{array}$ & $\begin{array}{r}92 \\
106\end{array}$ & $\begin{array}{l}104 \\
102\end{array}$ & $\begin{array}{r}116 \\
92\end{array}$ & \\
\hline 22 & Hip . . & . & C.3. & $\begin{array}{l}\text { S.R. } 135 \\
\text { B.P. } 114\end{array}$ & $\begin{array}{l}113 \\
108\end{array}$ & $\begin{array}{l}103 \\
110\end{array}$ & & & & & Died. \\
\hline 23 & Knee & . & C.3. & $\begin{array}{l}\text { S.R. } 118 \\
\text { B.P. } \quad-\end{array}$ & $\begin{array}{r}128 \\
-\end{array}$ & $\begin{array}{l}90 \\
-\end{array}$ & & & & & $\begin{array}{l}\text { Very much worse. } \\
\text { U.V.R. stopped. }\end{array}$ \\
\hline 24 & Hip . . & .. & C.3. & $\begin{array}{l}\text { S.R. } 102 \\
\text { B.P. } 111\end{array}$ & $\begin{array}{l}106 \\
112\end{array}$ & & & & & & $\begin{array}{l}\text { Worse. U.V.R. } \\
\text { stopped. }\end{array}$ \\
\hline 25 & Hip .. & . & C.3. & $\begin{array}{l}\text { S.R. } 165 \\
\text { B.P. } 114\end{array}$ & $\begin{array}{l}152 \\
106\end{array}$ & $\begin{array}{l}136 \\
111\end{array}$ & $\begin{array}{l}151 \\
116\end{array}$ & & & & No change. \\
\hline 26 & Hip .. & $\cdots$ & C.3. & $\begin{array}{lr}\text { S.R. } & 87 \\
\text { B.P. } & 105\end{array}$ & $\begin{array}{l}77 \\
99\end{array}$ & $\begin{array}{r}110 \\
95\end{array}$ & $\begin{array}{l}156 \\
108\end{array}$ & $\begin{array}{l}168 \\
108\end{array}$ & $\begin{array}{l}178 \\
120\end{array}$ & $\begin{array}{ll}160 & 184 \\
115 & 112\end{array}$ & Much improved. \\
\hline 27 & Hip .. & .. & C.3. & $\begin{array}{lr}\text { S.R. } & 82 \\
\text { B.P. } & 130\end{array}$ & $\begin{array}{r}66 \\
118\end{array}$ & $\begin{array}{r}80 \\
118\end{array}$ & $\begin{array}{r}67 \\
116\end{array}$ & $\begin{array}{r}70 \\
129\end{array}$ & $\begin{array}{r}70 \\
128\end{array}$ & $\begin{array}{rr}84 & 73 \\
128 & 121\end{array}$ & Worse. \\
\hline 28 & Spine & .. & C.2. & $\begin{array}{ll}\text { S.R. } 166 \\
\text { B.P. } 118\end{array}$ & $\begin{array}{l}187 \\
112\end{array}$ & $\begin{array}{l}190 \\
125\end{array}$ & $\begin{array}{l}190 \\
122\end{array}$ & $\begin{array}{l}193 \\
122\end{array}$ & $\begin{array}{l}196 \\
120\end{array}$ & $\begin{array}{ll}195 & 195 \\
130 & 134\end{array}$ & Improved. \\
\hline 29 & Knee & .. & C.2. & $\begin{array}{ll}\text { S.R. } 157 \\
\text { B.P. } 134\end{array}$ & $\begin{array}{l}154 \\
134\end{array}$ & $\begin{array}{l}136 \\
134\end{array}$ & $\begin{array}{l}130 \\
134\end{array}$ & & & & $\begin{array}{l}\text { Worse. L.V.R. } \\
\text { stopped. }\end{array}$ \\
\hline 30 & Spine & . & C.2. & $\begin{array}{lr}\text { S.R. } & 161 \\
\text { B.P. } & 92\end{array}$ & $\begin{array}{l}168 \\
100\end{array}$ & $\begin{array}{l}145 \\
102\end{array}$ & $\begin{array}{l}174 \\
104\end{array}$ & $\begin{array}{r}168 \\
98\end{array}$ & $\begin{array}{r}173 \\
98\end{array}$ & $\begin{array}{l}170 \\
100\end{array}$ & Improved. \\
\hline 31 & Hip .. & $\cdots$ & C.2. & $\begin{array}{ll}\text { S.R. } 184 \\
\text { B.P. } 104\end{array}$ & $\begin{array}{l}178 \\
106\end{array}$ & $\begin{array}{l}162 \\
106\end{array}$ & $\begin{array}{l}173 \\
110\end{array}$ & & & & No change. \\
\hline 32 & Hip .. & . & C.2. & $\begin{array}{ll}\text { S.R. } 182 \\
\text { B.P. } 112\end{array}$ & $\begin{array}{l}173 \\
112\end{array}$ & $\begin{array}{l}134 \\
115\end{array}$ & $\begin{array}{l}150 \\
114\end{array}$ & $\begin{array}{l}159 \\
115\end{array}$ & . & & Worse. \\
\hline 33 & Srine & .. & C.2. & $\begin{array}{l}\text { S.R. } 187 \\
\text { B.P. } 122\end{array}$ & $\begin{array}{l}183 \\
106\end{array}$ & $\begin{array}{l}189 \\
115\end{array}$ & $\begin{array}{l}188 \\
116\end{array}$ & $\begin{array}{l}183 \\
114\end{array}$ & & & No change. \\
\hline 34 & Hip .. & . & C.2. & $\begin{array}{l}\text { S.R. } 191 \\
\text { B.P. } \quad-\end{array}$ & $\begin{array}{r}190 \\
96\end{array}$ & $\begin{array}{r}196 \\
96\end{array}$ & $\begin{array}{r}189 \\
90\end{array}$ & $\begin{array}{l}196 \\
101\end{array}$ & $\begin{array}{r}195 \\
90\end{array}$ & $\begin{array}{l}197 \\
108\end{array}$ & Improved. \\
\hline 35 & $\mathrm{Hip}_{\text {... }}$ & .. & C.2. & $\begin{array}{ll}\text { S.R. } & 179 \\
\text { B.P. } & 100\end{array}$ & $\begin{array}{l}163 \\
106\end{array}$ & $\begin{array}{l}179 \\
109\end{array}$ & $\begin{array}{l}187 \\
121\end{array}$ & $\begin{array}{l}184 \\
112\end{array}$ & & & Improved. \\
\hline 36 & Spine & .. & C.2. & $\begin{array}{ll}\text { S.R. } & 192 \\
\text { B.P. } & 128\end{array}$ & $\begin{array}{l}188 \\
128\end{array}$ & $\begin{array}{l}188 \\
114\end{array}$ & $\begin{array}{l}191 \\
114\end{array}$ & $\begin{array}{l}191 \\
120\end{array}$ & $\begin{array}{l}195 \\
116\end{array}$ & $\begin{array}{ll}192 & 196 \\
112 & 124\end{array}$ & Improved. \\
\hline 37 & Hip .. & .. & C.1. & $\begin{array}{ll}\text { S.R. } & 170 \\
\text { B.P. } & 105\end{array}$ & $\begin{array}{r}187 \\
96\end{array}$ & $\begin{array}{l}191 \\
104\end{array}$ & $\begin{array}{l}195 \\
110\end{array}$ & $\begin{array}{l}193 \\
110\end{array}$ & & & Improved. \\
\hline 38 & Knee & . & C.1. & $\begin{array}{ll}\text { S.R. } & 190 \\
\text { B.P. } & 101\end{array}$ & $\begin{array}{l}191 \\
112\end{array}$ & $\begin{array}{l}193 \\
106\end{array}$ & $\begin{array}{l}193 \\
105\end{array}$ & $\begin{array}{l}191 \\
103\end{array}$ & - & i & Improved. \\
\hline 39 & Spine & .. & C.1. & $\begin{array}{l}\text { S.R. } \\
\text { B.P. }\end{array}$ & $\begin{array}{r}179 \\
-\end{array}$ & $\begin{array}{r}187 \\
-\end{array}$ & $\begin{array}{r}190 \\
-\end{array}$ & & & & Slightly improved. \\
\hline
\end{tabular}


TABLE I.-continued

\begin{tabular}{|c|c|c|c|c|c|c|c|c|c|c|c|c|}
\hline $\begin{array}{c}\text { Case } \\
\text { No. }\end{array}$ & Diagnosis. & Stage. & & nthly & $\begin{array}{r}\text { readi } \\
\text { and }\end{array}$ & $\begin{array}{l}\text { ngs of } \\
\text { blood }\end{array}$ & $\begin{array}{l}\text { sedim } \\
\text { press }\end{array}$ & $\begin{array}{l}\text { uentat } \\
\text { ure. }\end{array}$ & ion $\mathrm{ra}$ & & & Clinical progress. \\
\hline 40 & Spine $\quad \ldots$ & C.1. & $\begin{array}{lr}\text { S.R. } & 167 \\
\text { B.P. } & 94\end{array}$ & $\begin{array}{r}155 \\
98\end{array}$ & $\begin{array}{l}167 \\
102\end{array}$ & $\begin{array}{l}186 \\
105\end{array}$ & $\begin{array}{l}188 \\
112\end{array}$ & & & & & Improved. \\
\hline 41 & Peritonitis .. & - & $\begin{array}{ll}\text { S.R. } 195 \\
\text { B.P. } 100\end{array}$ & $\begin{array}{r}172 \\
92\end{array}$ & $\begin{array}{l}189 \\
110\end{array}$ & $\begin{array}{l}194 \\
110\end{array}$ & $\begin{array}{l}196 \\
118\end{array}$ & $\begin{array}{l}197 \\
128\end{array}$ & $\begin{array}{l}196 \\
122\end{array}$ & $\begin{array}{l}196 \\
122\end{array}$ & & Improved. \\
\hline 42 & Peritonitis .. & - & $\begin{array}{lr}\text { S.R. } & 166 \\
\text { B.P. } & 94\end{array}$ & $\begin{array}{r}170 \\
92\end{array}$ & $\begin{array}{r}163 \\
98\end{array}$ & $\begin{array}{r}177 \\
92\end{array}$ & $\begin{array}{l}190 \\
100\end{array}$ & $\begin{array}{l}186 \\
104\end{array}$ & $\begin{array}{l}190 \\
102\end{array}$ & $\begin{array}{l}190 \\
106\end{array}$ & & Improved. \\
\hline 43 & Peritonitis .. & - & $\begin{array}{lr}\text { S.R. } & 141 \\
\text { B.P. } & 80\end{array}$ & $\begin{array}{r}142 \\
80\end{array}$ & $\begin{array}{r}156 \\
88\end{array}$ & $\begin{array}{r}150 \\
98\end{array}$ & $\begin{array}{r}140 \\
95\end{array}$ & $\begin{array}{r}162 \\
96\end{array}$ & $\begin{array}{r}146 \\
92\end{array}$ & & & Improved. \\
\hline 44 & Peritonitis .. & - & $\begin{array}{ll}\text { S.R. } 188 \\
\text { B.P. } 120\end{array}$ & $\begin{array}{l}190 \\
116\end{array}$ & $\begin{array}{l}186 \\
116\end{array}$ & $\begin{array}{l}191 \\
117\end{array}$ & & & & & & Improved. \\
\hline 45 & Peritonitis .. & - & $\begin{array}{l}\text { S.R. } 192 \\
\text { B.P. }-\end{array}$ & $\begin{array}{r}198 \\
80\end{array}$ & $\begin{array}{r}198 \\
80\end{array}$ & $\begin{array}{r}198 \\
85\end{array}$ & $\begin{array}{r}198 \\
86\end{array}$ & & & & & Improved. \\
\hline 46 & Peritonitis .. & - & $\begin{array}{l}\text { S.R. } 123 \\
\text { B.P. }-\end{array}$ & $\begin{array}{r}187 \\
85\end{array}$ & $\begin{array}{r}194 \\
96\end{array}$ & $\begin{array}{l}194 \\
110\end{array}$ & $\begin{array}{l}194 \\
108\end{array}$ & $\begin{array}{l}195 \\
108\end{array}$ & $\begin{array}{l}192 \\
110\end{array}$ & & & Improved. \\
\hline 47 & $\begin{array}{l}\text { Cervical } \\
\text { Adenitis }\end{array}$ & - & $\begin{array}{l}\text { S.R. } 188 \\
\text { B.P. } \quad-\end{array}$ & $\begin{array}{l}192 \\
109\end{array}$ & $\begin{array}{l}198 \\
106\end{array}$ & $\begin{array}{l}197 \\
100\end{array}$ & $\begin{array}{l}198 \\
112\end{array}$ & $\begin{array}{l}195 \\
108\end{array}$ & $\begin{array}{l}196 \\
110\end{array}$ & $\begin{array}{l}196 \\
110\end{array}$ & & Improved. \\
\hline 48 & $\begin{array}{l}\text { Cervical } \\
\text { Adenitis }\end{array}$ & - & $\begin{array}{ll}\text { S.R. } & 196 \\
\text { B.P. } & 112\end{array}$ & $\begin{array}{l}188 \\
114\end{array}$ & $\begin{array}{l}193 \\
110\end{array}$ & $\begin{array}{l}195 \\
105\end{array}$ & & & & & & Improved. \\
\hline 49 & $\begin{array}{l}\text { Cervical } \\
\text { Adenitis }\end{array}$ & - & $\begin{array}{l}\text { S.R. } 194 \\
\text { B.P. } \quad-\end{array}$ & $\begin{array}{r}194 \\
86\end{array}$ & $\begin{array}{l}194 \\
100\end{array}$ & $\begin{array}{r}194 \\
98\end{array}$ & $\begin{array}{l}194 \\
100\end{array}$ & $\begin{array}{l}194 \\
101\end{array}$ & $\begin{array}{l}194 \\
100\end{array}$ & $\begin{array}{l}184 \\
102\end{array}$ & $\begin{array}{l}195 \\
106\end{array}$ & Improved. \\
\hline 50 & $\begin{array}{l}\text { Cervical } \\
\text { Adenitis }\end{array}$ & - & $\begin{array}{l}\text { S.R. } 160 \\
\text { B.P. } 110\end{array}$ & $\begin{array}{r}155 \\
98\end{array}$ & $\begin{array}{r}163 \\
96\end{array}$ & $\begin{array}{l}190 \\
118\end{array}$ & $\begin{array}{l}170 \\
108\end{array}$ & $\begin{array}{l}190 \\
112\end{array}$ & $\begin{array}{l}195 \\
116\end{array}$ & $\begin{array}{l}193 \\
116\end{array}$ & & Improved. \\
\hline 51 & $\begin{array}{l}\text { Cervical } \\
\text { Adenitis }\end{array}$ & - & $\begin{array}{l}\text { S.R. } 176 \\
\text { B.P. } 112\end{array}$ & $\begin{array}{l}134 \\
112\end{array}$ & $\begin{array}{l}170 \\
118\end{array}$ & $\begin{array}{l}180 \\
118\end{array}$ & & & & & & Improved. \\
\hline 53 & Ankle & - & $\begin{array}{ll}\text { S.R. } & 148 \\
\text { B.P. } & 130\end{array}$ & $\begin{array}{l}163 \\
122\end{array}$ & $\begin{array}{l}180 \\
115^{\prime}\end{array}$ & $\begin{array}{l}188 \\
135\end{array}$ & & & & & & Improved. \\
\hline 54 & Elbow & - & $\begin{array}{ll}\text { S.R. } & 180 \\
\text { B.P. } & 110\end{array}$ & $\begin{array}{l}194 \\
108\end{array}$ & $\begin{array}{l}195 \\
100\end{array}$ & $\begin{array}{l}194 \\
120\end{array}$ & $\begin{array}{l}192 \\
120\end{array}$ & $\begin{array}{l}192 \\
122\end{array}$ & $\begin{array}{l}195 \\
122\end{array}$ & & & Improved. \\
\hline 55 & Ankle $\quad \ldots$ & - & $\begin{array}{ll}\text { S.R. } & 170 \\
\text { B.P. } & 104\end{array}$ & $\begin{array}{r}185 \\
88\end{array}$ & $\begin{array}{l}188 \\
101\end{array}$ & $\begin{array}{l}181 \\
110\end{array}$ & & & & & & Improved. \\
\hline 56 & Epididymitis & - & $\begin{array}{ll}\text { S.R. } & 198 \\
\text { B.P. } & 128\end{array}$ & $\begin{array}{l}192 \\
114\end{array}$ & $\begin{array}{l}196 \\
122\end{array}$ & $\begin{array}{l}197 \\
113\end{array}$ & & & & & & No change. \\
\hline 57 & Scrofuladerma & - & $\begin{array}{l}\text { S.R. } 189 \\
\text { B.P. } \quad-\end{array}$ & $\begin{array}{r}176 \\
-\end{array}$ & $\begin{array}{r}191 \\
-\end{array}$ & $\begin{array}{r}191 \\
-\end{array}$ & & & & & & Improved. \\
\hline 58 & Lymphangitis & - & $\begin{array}{lr}\text { S.R. } & 191 \\
\text { B.P. } & 90\end{array}$ & $\begin{array}{r}192 \\
94\end{array}$ & $\begin{array}{l}197 \\
106\end{array}$ & $\begin{array}{l}194 \\
108\end{array}$ & $\begin{array}{l}194 \\
102\end{array}$ & & & & & Improved. \\
\hline 59 & Scrofuladerma & - & $\begin{array}{l}\text { S.R. } 196 \\
\text { B.P. } \quad-\end{array}$ & $\begin{array}{r}193 \\
90\end{array}$ & $\begin{array}{r}195 \\
95\end{array}$ & $\begin{array}{r}196 \\
94\end{array}$ & $\begin{array}{r}197 \\
92\end{array}$ & $\begin{array}{l}192 \\
102\end{array}$ & $\begin{array}{r}195 \\
95\end{array}$ & & & Improved. \\
\hline 60 & Kidney & - & $\begin{array}{ll}\text { S.R. } & 157 \\
\text { B.P. } & -\end{array}$ & $\begin{array}{r}184 \\
-\end{array}$ & $\begin{array}{r}185 \\
-\end{array}$ & $\begin{array}{c}195 \\
--\end{array}$ & $\begin{array}{r}196 \\
-\end{array}$ & 196 & $\begin{array}{r}195 \\
-\end{array}$ & $\begin{array}{r}192 \\
-\end{array}$ & & Improved. \\
\hline
\end{tabular}


TABLE II.

Series of Control Cases.

\begin{tabular}{|c|c|c|c|c|c|c|c|c|c|c|}
\hline $\begin{array}{l}\text { Case } \\
\text { No. }\end{array}$ & Diagnosis & & Stage. & & onthly & $\begin{array}{l}\text { eadin } \\
\text { and }\end{array}$ & $\begin{array}{l}\text { of sec } \\
\text { od pr }\end{array}$ & $\begin{array}{l}\text { urentat } \\
\text { ure. }\end{array}$ & rate & \\
\hline 1 & Right Hip & $\ldots$ & C.2. & $\begin{array}{l}\text { S.R. } \\
\text { B.P. }\end{array}$ & $\begin{array}{r}193 \\
88\end{array}$ & $\begin{array}{l}192 \\
108\end{array}$ & $\begin{array}{r}193 \\
92\end{array}$ & $\begin{array}{r}193 \\
94\end{array}$ & $\begin{array}{r}196 \\
90\end{array}$ & $\begin{array}{r}197 \\
90\end{array}$ \\
\hline 2 & Hip ... & & C.2. & $\begin{array}{l}\text { S.R. } \\
\text { B.P. }\end{array}$ & $\begin{array}{l}168 \\
100\end{array}$ & $\begin{array}{l}160 \\
100\end{array}$ & $\begin{array}{l}156 \\
106\end{array}$ & $\begin{array}{l}164 \\
101\end{array}$ & $\begin{array}{l}157 \\
102\end{array}$ & $\begin{array}{l}172 \\
110\end{array}$ \\
\hline 3 & Hip ... & $\ldots$ & C.2. & $\begin{array}{l}\text { S.R. } \\
\text { B.P. }\end{array}$ & $\begin{array}{l}175 \\
108\end{array}$ & $\begin{array}{l}188 \\
110\end{array}$ & $\begin{array}{l}190 \\
110\end{array}$ & $\begin{array}{l}188 \\
112\end{array}$ & $\begin{array}{l}191 \\
108\end{array}$ & $\begin{array}{l}181 \\
111\end{array}$ \\
\hline 4 & Hip ... & $\ldots$ & C.1. & $\begin{array}{l}\text { S.R. } \\
\text { B.P. }\end{array}$ & $\begin{array}{r}196 \\
98\end{array}$ & $\begin{array}{l}196 \\
104\end{array}$ & $\begin{array}{l}197 \\
104\end{array}$ & $\begin{array}{r}190 \\
98\end{array}$ & $\begin{array}{l}185 \\
105\end{array}$ & $\begin{array}{l}196 \\
124\end{array}$ \\
\hline 5 & Ankle & $\ldots$ & - & $\begin{array}{l}\text { S.R. } \\
\text { B.P. }\end{array}$ & $\begin{array}{l}153 \\
105\end{array}$ & $\begin{array}{l}166 \\
110\end{array}$ & $\begin{array}{l}168 \\
108\end{array}$ & $\begin{array}{l}183 \\
110\end{array}$ & $\begin{array}{l}168 \\
107\end{array}$ & $\begin{array}{l}174 \\
116\end{array}$ \\
\hline 6 & Osteitis & $\ldots$ & - & $\begin{array}{l}\text { S.R. } \\
\text { B.P. }\end{array}$ & $\begin{array}{l}180 \\
118\end{array}$ & $\begin{array}{l}178 \\
114\end{array}$ & $\begin{array}{l}179 \\
116\end{array}$ & $\begin{array}{l}178 \\
118\end{array}$ & $\begin{array}{l}179 \\
119\end{array}$ & $\begin{array}{l}180 \\
122\end{array}$ \\
\hline 7 & Spine & $\ldots$ & C.2. & $\begin{array}{l}\text { S.R. } \\
\text { B.P. }\end{array}$ & $\begin{array}{r}196 \\
95\end{array}$ & $\begin{array}{r}194 \\
90\end{array}$ & $\begin{array}{r}195 \\
90\end{array}$ & $\begin{array}{r}195 \\
96\end{array}$ & $\begin{array}{r}196 \\
98\end{array}$ & $\begin{array}{l}197 \\
100\end{array}$ \\
\hline 8 & Hip ... & $\ldots$ & C.3. & $\begin{array}{l}\text { S.R. } \\
\text { B.P. }\end{array}$ & $\begin{array}{r}74 \\
116\end{array}$ & $\begin{array}{r}73 \\
112\end{array}$ & $\begin{array}{l}124 \\
116\end{array}$ & $\begin{array}{l}156 \\
118\end{array}$ & $\begin{array}{l}178 \\
110\end{array}$ & $\begin{array}{l}180 \\
112\end{array}$ \\
\hline 9 & Osteitis & $\ldots$ & - & $\begin{array}{l}\text { S.R. } \\
\text { B.P. }\end{array}$ & $\begin{array}{l}187 \\
100\end{array}$ & $\begin{array}{l}190 \\
102\end{array}$ & $\begin{array}{l}187 \\
100\end{array}$ & $\begin{array}{r}189 \\
90\end{array}$ & $\begin{array}{l}188 \\
102\end{array}$ & $\begin{array}{l}186 \\
104\end{array}$ \\
\hline 10 & Hip ... & $\ldots$ & C.2. & $\begin{array}{l}\text { S.R. } \\
\text { B.P. }\end{array}$ & $\begin{array}{l}181 \\
120\end{array}$ & $\begin{array}{l}189 \\
124\end{array}$ & $\begin{array}{l}189 \\
122\end{array}$ & $\begin{array}{l}190 \\
124\end{array}$ & $\begin{array}{l}182 \\
120\end{array}$ & $\begin{array}{l}190 \\
118\end{array}$ \\
\hline 11 & Spine & $\ldots$ & C.3. & $\begin{array}{l}\text { S.R. } \\
\text { B.P. }\end{array}$ & $\begin{array}{l}112 \\
110\end{array}$ & $\begin{array}{l}174 \\
122\end{array}$ & $\begin{array}{l}177 \\
120\end{array}$ & $\begin{array}{l}182 \\
128\end{array}$ & $\begin{array}{l}195 \\
132\end{array}$ & $\begin{array}{l}189 \\
132\end{array}$ \\
\hline 12 & Knee & $\ldots$ & C.1. & $\begin{array}{l}\text { S.R. } \\
\text { B.P. }\end{array}$ & $\begin{array}{r}197 \\
115\end{array}$ & $\begin{array}{l}195 \\
114\end{array}$ & $\begin{array}{l}193 \\
106\end{array}$ & $\begin{array}{l}194 \\
120\end{array}$ & $\begin{array}{l}190 \\
115\end{array}$ & $\begin{array}{l}192 \\
120\end{array}$ \\
\hline
\end{tabular}


TABLE II.-continned.

\begin{tabular}{|c|c|c|c|c|c|c|c|c|c|c|}
\hline \multirow{2}{*}{$\begin{array}{c}\begin{array}{c}\text { Case } \\
\text { No. }\end{array} \\
13\end{array}$} & \multicolumn{2}{|c|}{ Diagnosis. } & \multirow{2}{*}{ Stage. } & \multicolumn{7}{|c|}{$\begin{array}{l}\text { Monthly readings of sedimentation rates } \\
\text { and blood pressure. }\end{array}$} \\
\hline & Ankle & $\cdots$ & & $\begin{array}{l}\text { S.R. } \\
\text { B.P. }\end{array}$ & $\begin{array}{r}136 \\
98\end{array}$ & $\begin{array}{l}146 \\
108\end{array}$ & $\begin{array}{l}160 \\
102\end{array}$ & $\begin{array}{l}159 \\
108\end{array}$ & $\begin{array}{l}170 \\
112\end{array}$ & $\begin{array}{l}164 \\
107\end{array}$ \\
\hline 14 & Osteitis & $\cdots$ & - & $\begin{array}{l}\text { S.R. } \\
\text { B.P. }\end{array}$ & $\begin{array}{l}187 \\
102\end{array}$ & $\begin{array}{r}192 \\
96\end{array}$ & $\begin{array}{r}191 \\
96\end{array}$ & $\begin{array}{l}188 \\
106\end{array}$ & & \\
\hline 15 & Osteitis & $\ldots$ & - & $\begin{array}{l}\text { S.R. } \\
\text { B.P. }\end{array}$ & $\begin{array}{l}168 \\
100\end{array}$ & $\begin{array}{l}168 \\
108\end{array}$ & $\begin{array}{l}178 \\
105\end{array}$ & $\begin{array}{l}180 \\
106\end{array}$ & & \\
\hline 16 & Spine & $\ldots$ & C.2. & $\begin{array}{l}\text { S.R. } \\
\text { B.P. }\end{array}$ & $\begin{array}{l}190 \\
102\end{array}$ & $\begin{array}{l}193 \\
102\end{array}$ & $\begin{array}{r}190 \\
92\end{array}$ & $\begin{array}{l}193 \\
100\end{array}$ & $\begin{array}{l}193 \\
102\end{array}$ & $\begin{array}{l}195 \\
108\end{array}$ \\
\hline 17 & Hip ... & $\ldots$ & C.1. & $\begin{array}{l}\text { S.R. } \\
\text { B.P. }\end{array}$ & $\begin{array}{l}197 \\
112\end{array}$ & $\begin{array}{l}198 \\
112\end{array}$ & $\begin{array}{l}198 \\
114\end{array}$ & $\begin{array}{l}197 \\
115\end{array}$ & $\begin{array}{l}198 \\
120\end{array}$ & \\
\hline 18 & Hip ... & $\ldots$ & C.1. & $\begin{array}{l}\text { S.R. } \\
\text { B.P. }\end{array}$ & $\begin{array}{l}189 \\
104\end{array}$ & $\begin{array}{l}194 \\
114\end{array}$ & $\begin{array}{l}193 \\
108\end{array}$ & $\begin{array}{l}192 \\
105\end{array}$ & $\begin{array}{l}187 \\
110\end{array}$ & $\begin{array}{l}170 \\
114\end{array}$ \\
\hline 19 & Spine & $\ldots$ & C.3. & $\begin{array}{l}\text { S.R. } \\
\text { B.P. }\end{array}$ & $\begin{array}{r}160 \\
82\end{array}$ & $\begin{array}{l}142 \\
102\end{array}$ & $\begin{array}{l}153 \\
104\end{array}$ & $\begin{array}{l}151 \\
104\end{array}$ & $\begin{array}{l}184 \\
112\end{array}$ & $\begin{array}{r}170 \\
95\end{array}$ \\
\hline 20 & Hip ... & $\ldots$ & C.2. & $\begin{array}{l}\text { S.R. } \\
\text { B.P. }\end{array}$ & $\begin{array}{l}192 \\
100\end{array}$ & $\begin{array}{l}192 \\
115\end{array}$ & $\begin{array}{l}193 \\
114\end{array}$ & $\begin{array}{l}195 \\
112\end{array}$ & $\begin{array}{l}194 \\
110\end{array}$ & $\begin{array}{l}197 \\
104\end{array}$ \\
\hline 21 & Knee & $\cdots$ & C.1. & $\begin{array}{l}\text { S.R. } \\
\text { B.P. }\end{array}$ & $\begin{array}{l}192 \\
128\end{array}$ & $\begin{array}{l}191 \\
130\end{array}$ & $\begin{array}{l}191 \\
126\end{array}$ & $\begin{array}{l}186 \\
128\end{array}$ & $\begin{array}{l}192 \\
122\end{array}$ & \\
\hline 22 & Ankle & $\ldots$ & - & $\begin{array}{l}\text { S.R. } \\
\text { B.P. }\end{array}$ & $\begin{array}{l}169 \\
102\end{array}$ & $\begin{array}{l}179 \\
102\end{array}$ & $\begin{array}{l}176 \\
102\end{array}$ & $\begin{array}{l}170 \\
104\end{array}$ & & \\
\hline 23 & Elbow & $\cdots$ & - & $\begin{array}{l}\text { S.R. } \\
\text { B.P. }\end{array}$ & $\begin{array}{l}180 \\
108\end{array}$ & $\begin{array}{l}189 \\
104\end{array}$ & $\begin{array}{l}188 \\
104\end{array}$ & $\begin{array}{l}191 \\
108\end{array}$ & $\begin{array}{l}190 \\
103\end{array}$ & \\
\hline 24 & Osteitis & $\ldots$ & - & $\begin{array}{l}\text { S.R. } \\
\text { B.P. }\end{array}$ & $\begin{array}{l}171 \\
118\end{array}$ & $\begin{array}{l}160 \\
110\end{array}$ & $\begin{array}{l}158 \\
110\end{array}$ & $\begin{array}{l}153 \\
112\end{array}$ & $\begin{array}{l}165 \\
109\end{array}$ & $\begin{array}{l}170 \\
110\end{array}$ \\
\hline 25 & Hip ... & $\cdots$ & C.2. & $\begin{array}{l}\text { S.R. } \\
\text { B.P. }\end{array}$ & $\begin{array}{l}164 \\
132\end{array}$ & $\begin{array}{l}167 \\
122\end{array}$ & $\begin{array}{l}148 \\
124\end{array}$ & $\begin{array}{l}172 \\
122\end{array}$ & $\begin{array}{l}150 \\
128\end{array}$ & \\
\hline
\end{tabular}


In all, some 469 monthly examinations are recorded in these notes. Conclusions.

1. A series of sedimentation rates has a definite place and value in forming an opinion as to the progress and prognosis of tuberculosis in children.

2. As a diagnostic agent its scope is limited. In this work may be found a few cases of undoubted tuberculosis which have a normal sedimentation rate. An abnormal rate is probably always an indication of disease, not necessarily tuberculosis.

3. It is felt that extremes of temperature may alter the reading to some extent. For this reason the room temperature was noted. The temperature of the room in which the readings were taken was almost always between $55^{\circ}$ and $60^{\circ} \mathrm{F}$.

4. It is considered that a series of sedimentation rates may be of great value in controlling the dosage and the duration of treatment by ultra-violet radiation.

\section{The Blood Pressure.}

The instrument used was a desk model Baumanometer. The residual systolic pressure was estimated by finger palpation as it was found that the auscultatory method was not practicable in children.

The readings were taken at rest, between exposures to ultra-violet radiation, and at approximately the same time after a meal.

It is to be admitted that in children there is a certain difficulty about the taking of blood pressure, but it was found that after due consideration had been given to this fact there were certain tendencies which could not be disregarded.

The taking of the blood pressure was at first regarded solely in the rôle of a routine examination, but as the number of readings increased the following impressions were gleaned :-

(1) That in many cases which were clinically and by the sedimentation rate showing marked improvement, the blood pressure rose in a correspondingly definite fashion. Briefly the tendency in cases showing improvement was for the blood pressure to rise. This was also shown in some of the control cases on ordinary routine open-air treatment.

(2) That following one month's ultra-violet radiation certain cases show a definite fall in blood pressure, and that this often coincided with the fall in the sedimentation rate reading which was referred to as the 'negative phase.'

(3) That the variations shown in the blood-pressure readings were more marked in those cases receiving radiation than in the control cases. 
III. OBSERVATIONS ON ENDOCRINE ACTIVITY \& CARBOHYDRATE TOLERANCE IN CONNECTION WITH ULTRA-VIOLET RADIATION.

During the period that an investigation was being made into the value of ultra-violet radiation in the treatment of tuberculous children, it was observed that in certain cases the reaction to light suggested the possibility of an association between ultra-violet radiation and the endocrine glands.

The relationship between low blood pressure and pigmentation, in particular, gave rise to the premise that the suprarenal glands play more than a passive part in the action of light on the body.

On the strength of these observations, and also upon certain other evidence gleaned from various sources, it was decided to ascertain what effect, if any, the exhibition of suprarenal extract might have on one of these cases. The first case (and for a time the only case) so treated was that of a boy of fourteen who had been on a frame for four years, during the latter part of which he had been receiving ultra-violet radiation. When suprarenal therapy was begun he was almost in extremis : there was widespread odema up to the axillæ, the hip was discharging from twelve sinuses and the buttock was partially gangrenous, the liver and spleen were much enlarged and the urine was loaded with albumen. There was deep brown pigmentation on the whole body. The Wassermann reaction was negative. The blood pressure was $100 \mathrm{~mm}$. and the sedimentation rate was $119 \mathrm{~mm}$. Ultra-violet radiation had been stopped about a month previously, but was re-started when treatment by suprarenal extract was begun. Some eight months later the boy presented quite a different picture : the pigmentation was no longer excessive, the liver though palpable was now shrunken and the spleen was not palpable. There was a trace of albumen in the urine, the B.P. was $130 \mathrm{~mm}$., and the sedimentation rate $138 \mathrm{~mm}$. Since then the improvement has continued, and the patient is now able to get up though he is still an in-patient in the Hospital.

When it was seen that improvement was taking place in this case, other patients were similarly treated, but unfortunately the results in the series of cases were not so encouraging. Following upon this line of thought it was considered that it might help if the efficiency of these glands could be estimated. No reliable method of estimating this efficiency was found in the literature. As it is known that the suprarenal glands influence carbohydrate metabolism, it was thought that it might be of interest to ascertain the patients' carbohydrate tolerance before and after a period of ultra-violet radiation.

The conclusions and technique are embodied in the following paragraphs.

The Adrenals and Light.

The hypothesis enunciated in the introduction, namely, that the adrenal glands play more than a passive part in the treatment of disease by ultra-violet radiation, was based upon certain observations in the cases under investigation. and upon other evidence extracted from the literature. 
TABLE III.

C'ases Treated with Suprarenal and Parathyoid Extracts in conjunction WITH U.V. RADiation.

\begin{tabular}{|c|c|c|c|c|c|c|c|c|c|c|c|c|c|}
\hline \multirow{2}{*}{$\begin{array}{l}\text { Case } \\
\text { No. } \\
11\end{array}$} & \multicolumn{2}{|c|}{ Diagnosis. } & \multirow{2}{*}{$\begin{array}{c}\text { Stage. } \\
\text { C.3. }\end{array}$} & \multicolumn{9}{|c|}{$\begin{array}{l}\text { Monthly readings of sedimentation rate } \\
\text { and blood pressure. }\end{array}$} & \multirow{2}{*}{$\begin{array}{l}\text { Clinical progress. } \\
\text { Much improved. }\end{array}$} \\
\hline & Hip .. & .. & & S.R. 119 & $\begin{array}{l}74 \\
99\end{array}$ & 79* & 71 & 99 & 102 & $\begin{array}{r}90 \\
199\end{array}$ & 101 & $\begin{array}{ll}121 & 138\end{array}$ & \\
\hline 12 & Spine & .. & C.3. & $\begin{array}{l}\text { S.R. } 154^{*} \\
\text { B.P. } 114\end{array}$ & $\begin{array}{l}118 \\
102\end{array}$ & $\begin{array}{l}148 \\
108\end{array}$ & $\begin{array}{l}170 \\
112\end{array}$ & & & & & & Worse. \\
\hline 13 & Spine & .. & C.3. & $\begin{array}{l}\text { S.R. } 123 \\
\text { B.P. } 92\end{array}$ & $\begin{array}{l}120 \\
104\end{array}$ & $\begin{array}{l}95^{*} \\
88\end{array}$ & $\begin{array}{l}107 \\
112\end{array}$ & $\begin{array}{l}106 \\
100\end{array}$ & $\begin{array}{r}111 \\
92\end{array}$ & $\begin{array}{r}96 \\
104\end{array}$ & $\begin{array}{r}93 \\
102\end{array}$ & $\begin{array}{r}54 \\
102\end{array}$ & No change. \\
\hline 14 & Peritonitis & . & - & $\begin{array}{l}\text { S.R. } 154 \\
\text { B.P. } \quad 96\end{array}$ & $\begin{array}{l}140 \\
100\end{array}$ & $\begin{array}{l}101 \\
100\end{array}$ & $\begin{array}{l}101^{*} \\
106\end{array}$ & $\begin{array}{l}134 \\
108\end{array}$ & $\begin{array}{l}116 \\
112\end{array}$ & $\begin{array}{l}102 \\
108\end{array}$ & $\begin{array}{l}114 \\
108\end{array}$ & & No change . \\
\hline 15 & Hip & .. & C.3. & $\begin{array}{l}\text { S.R. } 132 \\
\text { B.P. } 108\end{array}$ & $\begin{array}{l}107 \\
110\end{array}$ & $\begin{array}{l}145 \\
122\end{array}$ & $\begin{array}{l}141 \\
126\end{array}$ & $\begin{array}{l}142^{*} \\
126\end{array}$ & $\begin{array}{l}130 \\
122\end{array}$ & $\begin{array}{l}159 \\
120\end{array}$ & $\begin{array}{l}182 \\
126\end{array}$ & $\begin{array}{l}168 \\
124\end{array}$ & Improved. \\
\hline 16 & Osteitis & .. & C.3. & $\begin{array}{l}\text { S.R. } 148 \\
\text { B.P. } 84\end{array}$ & $\begin{array}{l}79 \\
75\end{array}$ & $\begin{array}{l}83^{*} \\
80\end{array}$ & $\begin{array}{r}100 \\
92\end{array}$ & $\begin{array}{r}110 \\
94\end{array}$ & $\begin{array}{r}100 \\
92\end{array}$ & $\begin{array}{l}90 \\
95\end{array}$ & 98 & 76 & No change \\
\hline 17 & Hip .. & .. & C.3. & $\begin{array}{l}\text { S.R. } 133 \\
\text { B.P. } \quad-\end{array}$ & $\begin{array}{l}85 \\
94\end{array}$ & $\begin{array}{l}117 \\
108\end{array}$ & $\begin{array}{l}75 \\
90\end{array}$ & $\begin{array}{l}91 * \\
96\end{array}$ & $\begin{array}{l}118 \\
104\end{array}$ & $\begin{array}{r}81 \\
114\end{array}$ & $\begin{array}{r}61 \\
116\end{array}$ & & No change. \\
\hline 18 & Hip .. & . & C.3. & $\begin{array}{l}\text { S.R. } 124 \\
\text { B.P. } 126\end{array}$ & $\begin{array}{l}122 \\
118\end{array}$ & $\begin{array}{l}120^{*} \\
110\end{array}$ & $\begin{array}{l}141 \\
102\end{array}$ & $\begin{array}{l}133 \\
108\end{array}$ & $\begin{array}{l}130 \\
116\end{array}$ & $\begin{array}{l}136 \\
122\end{array}$ & $\begin{array}{l}131 \\
122\end{array}$ & & Improved. \\
\hline 19 & Spine & . & C.3. & $\begin{array}{lr}\text { S.R. } & 94 \\
\text { B.P. } & 102\end{array}$ & $\begin{array}{r}85^{*} \\
107\end{array}$ & $\begin{array}{r}78 \\
115\end{array}$ & & & & & & & Worse. \\
\hline 20 & Peritonitis & $\cdot$ & 一 & $\begin{array}{rr}\text { S.R. } & 178 \\
\text { B.P. } & 92\end{array}$ & $\begin{array}{r}173 \\
90\end{array}$ & $\begin{array}{c}155^{*} \\
96\end{array}$ & $\begin{array}{l}172 \\
112\end{array}$ & $\begin{array}{l}188 \\
110\end{array}$ & $\begin{array}{l}180 \\
108\end{array}$ & & & & Improved. \\
\hline
\end{tabular}

* Denotes beginning of endocrine therapy.

It is suggested that it may be accepted that the visible reaction to light is in the form of a protective pigmentation, and that in suprarenal deficiency there is also present in most cases a pigmentation, although as far as is understood at present there is no evidence to show that these pigments are of an exactly similar nature. The original observation which gave rise to the hypothesis was that certain cases were deeply pigmented, although the disease was progressive, and in many cases the patients were in danger of developing amyloid disease if they had not already done so.

That pigmentation of itself is no criterion in prognosis is shown by the following extracts :-

(a) Rost ${ }^{8}$ and others believe that pigmentation is an undesirable element formed by light radiation in the treatment of tuberculosis. 
(b) Mayer ${ }^{9}$ states that the sudden development in patients of the capacity to produce pigment may often be associated with the rapid cure of a new tuberculous focus, strongly in contrast with the previous slow healing of an area of disease when the patient did not pigment. Yet frequently patients who have developed marked pigmentation fail to heal their disease.

It was found on further investigation that this particular type of case, the progressive, pigmented and often amyloid patient, had in most instances although not in all, a comparatively low blood pressure. (The question of rajsed blood pressure with albuminuria is also to be considered in these cases, this majority of which had albuminuria.)

Perhaps a much more significant factor was the initial fall in blood pressure following the first period (one month) of artificial radiation, and that this was often accompanied by a corresponding fall in the sedimentation-rate reading. When this was compared with the fact that in those cases which were showing improvement, the blood pressure tended to rise after the first month, it was wondered whether that secretion in the body best known to be capable of raising blood pressure was associated with these changes.

It has been observed by Mayer and Bell Ferguson ${ }^{12}$ that the immediate effect of a light bath is to produce a lowering of the blood pressure, which effect normally disappears within a short time after exposure. This may be explained by a vaso-dilatation of the surface capillaries, and to what extent the sympathetic nervous system could in itself be associated with any of the observations recorded in these notes it would be difficult to say.

In 'sunstroke,' which corresponds to an overdose of sunlight, it is found that the blood pressure remains low. There would appear to be a similarity between sunstroke and surgical shock, and in the latter Crile strongly advocates the use of adrenalin. Dale and Richards ${ }^{13}$ have shown recently that adrenalin is able to act directly without being dependent upon other substances, e.g., histamine.

The thought which presents itself is whether the action of adrenalin is that of supplying a physiological want.

Another condition which simulates surgical shock is death in an animal following an injection of hæmatoporphyrin. Gilchrist ${ }^{14}$ has stated that investigations into hæmatoporphyrinuria has revealed that the cause of death was the depression of blood pressure and dilatation of vessels. That there is in cases of hæmatoporphyrinuria an extreme 'light sensitiveness.' has been shown by Ashby ${ }^{20}$ in describing a patient who suffered from attacks of Hydroa whenever the weather was hot and sunny and who was very pigmented and sunburnt. Betz ${ }^{15}$ injected into himself $0.2 \mathrm{grm}$. hæmatoporphyrin and became extremely sensitive to light. Here may be mentioned a case (No. 20) in this series where following two periods of exposure to ultra-violet radiation, with a week's rest in between, there developed on each occasion an erythematous scaly eruption, which disappeared and did not return following the exhibition 
of suprarenal extract, the exposure being continued at the same time. Is it reasonable to suggest that in adrenalin may possibly be found a desensitizing agent ?

The following paragraph is from Bell Ferguson's book on the Quartz $\operatorname{lamp}^{16}:-$

Dr. F. Talbot, in a letter to the British Medical Journal, relates how an asthmatical boy was given an ultra-violet radiation bath, and also his brother who was not asthmatical. Within some hours the first mentioned developed an attack of asthma and showed a much milder erythema than his brother. He was given adrenalin. Within half an hour the asthma passed off and $3 \frac{1}{2}$ hours later it was observed that his erythema had increased and was now almost as marked as in the case of his brother. Dr. Talbot suggests that the skin's reaction to light makes a demand on the adrenals which in this case temporarily exhausted them and induced an attack of asthma, and that the dose of adrenalin restored the balance and enabled the skin reaction to be completed.

Another aspect of the hypothesis is that of the 'antitoxic' property of the suprarenal capsules which has been suggested by Schafer ${ }^{11}$. He states that enlargement of the suprarenal capsules, mainly of the cortex, has been frequently noticed in infection and as the result of inoculation with disease toxins. As all cases which were treated with suprarenal extract were complicated by a secondary pyogenic infection, the question arises whether the improvement, if any, could be due to some antitoxic action of the suprarenal extract. But if this is true the fact remains that these cases do badly on artificial light and that this might be due to some further adrenal drain.

The addition of parathyroid extract, although empirical, was considered advisable for the following reasons :-

(1) It has been suggested that endocrine extracts have a greater action in combination with one another, and that the parathyroid gland is the compliment of the suprarenal gland ${ }^{17}$.

(2) It is known that following a long fixation on frames decalcification occurs, and that this takes place in the neighbourhood of a tuberculous joint. It was considered that the addition of parathyroid extract might assist in mobilizing the calcium content of the body and also in counteracting infection.

(3) Richter ${ }^{18}$ suggests that in Addison's disease there is an increased breaking down of bone.

(4) Rabbits exposed to ultra-violet radiation develop a hypertrophy of the parathyroid glands ${ }^{19}$.

It was felt that the injection of adrenalin into the skin was not justified. For that reason and also that perhaps the whole gland would be more likely to produce an action, the dessicated adrenal substance (grn. 2 in gelatine capsules) was used throughout with parathyroid tablets, grn. $\frac{1}{2} \sigma$ (Messrs. Parke, Davis \& Co.). 


\section{Conclusions.}

(1) That there has been a therapeutic action in a few cases following the exhibition of these extracts is supported by the following evidence :-

(a) It would appear that the blood pressure has been raised appreciably in some of the cases. (Leyton ${ }^{21}$ states that adrenal extract, although when administered by mouth it fails ordinarily to produce elevation of blood pressure, will bring about this effect in cases of Addison's disease.) Two control cases did not show any decided rise following a course-two months-of suprarenal extract.

(b) That the pigmentation in some cases has decidedly diminished in degree following treatment on these lines. (Allowance is made for the fact that some of these cases began treatment in the summer.)

(2) It is too early to weigh up how much improvement has been shown, but when it is remembered that these cases were advanced, and that some of them had entered that last stage which usually terminates in death, it is felt that there has been a definite response in the way desired.

(3) No conclusion regarding the influence of ultra-violet radiation upon the action of the extracts has been made, but it would appear that any change has been independent of the former.

(4) The observations although inconclusive would, it is thought, repay further investigation. It is felt that the particular fields in which trial might be made are: (a) Cases verging on amyloid disease ; $(b)$ Cases sensitive to ultra-violet radiation.

\section{Ultra-Violet Radiation and Carbohydrate Metabolism.}

Interest in the adrenal glands having been aroused the question of whether it were possible to estimate their efficiency presented itself. There appears to be no satisfactory method of doing this.

Sergent ${ }^{22}$ has described the appearance of a white line, la ligne blanche surrénale, following the stroking of the skin in a case of adrenal insufficiency. This was tried and although it was produced in some of the cases suspected of being deficient in adrenal secretion, it was to an almost equal extent present in many other cases.

It has been shown ${ }^{23}$ that an injection of adrenalin produces an increase in the blood sugar and that in Addison's disease the sugar tolerance is increased.

It was felt that it might be of interest to see what effect, if any, a series of exposures to ultra-violet radiation would have on the carbohydrate tolerance. It was thought that, if it were true that light stimulated the formation of, and in some way used up the adrenal secretion, a change might be found in the 
sugar-tolerance curves before and after a course of exposures. It was assumed also that any results could not be conclusive, for a stimulus applied to the adrenals probably affects the complete endocrine system, and that any change could be associated with other hormones, as for example, the thyroid or pancreas.

Here may be mentioned the case of an adult female to whom it was suggested by an outside practitioner that a course of ultra-violet radiation might be of benefit to her. The diagnosis was that of nervous breakdown, but after about six weeks' treatment she developed marked exophthalmos, and although the thyroid gland was enlarged to begin with it became very much bigger.

It was decided to use a large dose of glucose so that any variations in the curves would be more evident, and for this reason a comparatively large amount for children ( $80 \mathrm{grm}$.) was used. It has been stated that, however large the amount of glucose given, the blood sugar will not rise any higher than if the

Chart of Average Blool, Sugar Curvis.
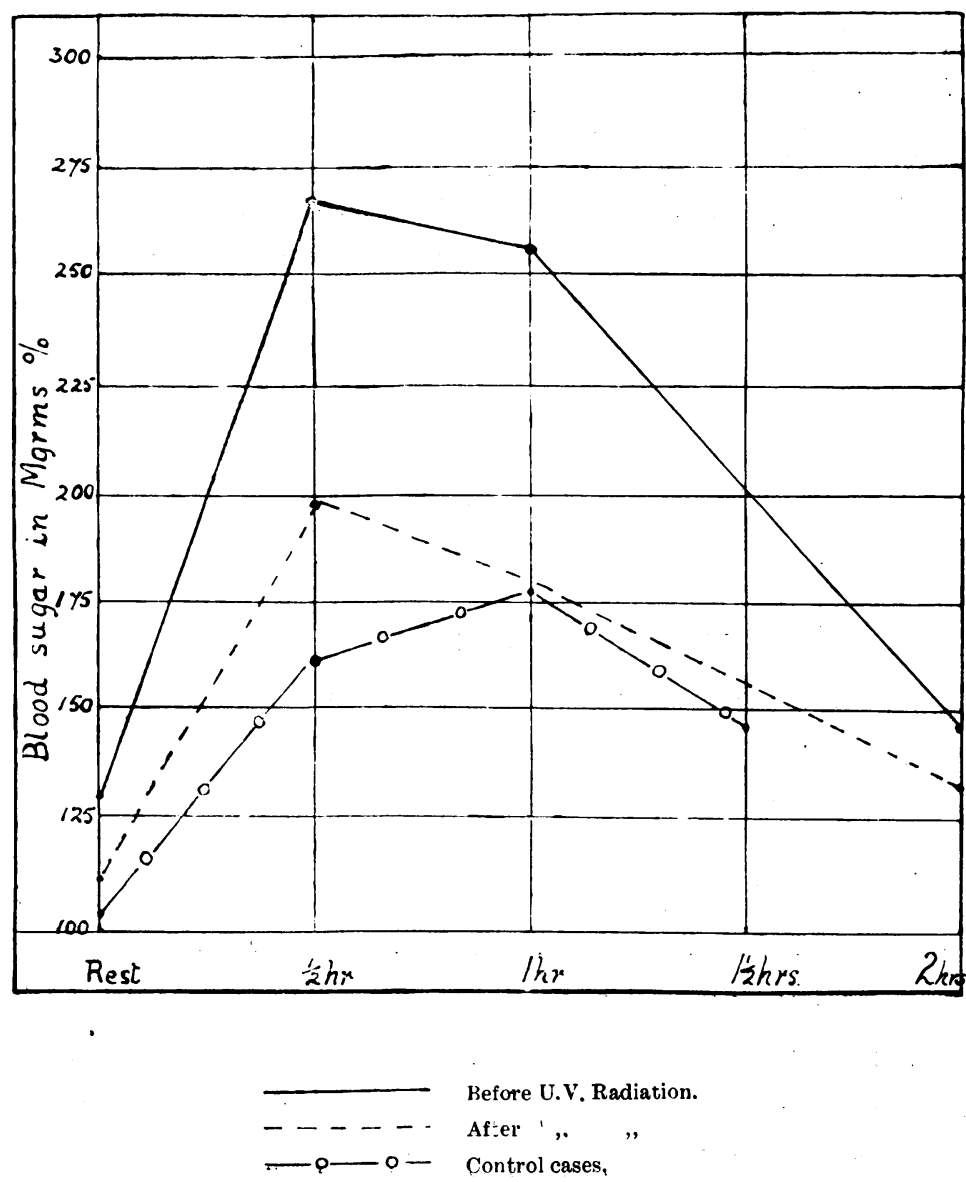
TABLE IV.

Carbohydrate Tolerance and Ultra-Violet Radiation Results.

\begin{tabular}{|c|c|c|c|c|c|c|c|}
\hline \multirow{2}{*}{ Case No. } & \multicolumn{7}{|c|}{ Blood sugar : mgrm. per cent. } \\
\hline & & & Resting & $\frac{1}{2}$ hour. & 1 hour. & $1 \frac{1}{2}$ hours. & 2 hours. \\
\hline \multirow[t]{2}{*}{1} & Before & $\ldots$ & 145 & 353 & 376 & - & 162 \\
\hline & After & $\ldots$ & 118 & 221 & 154 & - & 149 \\
\hline \multirow[t]{2}{*}{2} & Before & $\ldots$ & 142 & 289 & 317 & - & 172 \\
\hline & After & $\ldots$ & 97 & 194 & 146 & - & 155 \\
\hline \multirow[t]{2}{*}{3} & Before & $\ldots$ & 105 & 238 & 219 & 217 & - \\
\hline & After & $\ldots$ & 141 & 210 & 205 & 165 & 84 \\
\hline \multirow[t]{2}{*}{4} & Before & $\ldots$ & 141 & 257 & 253 & - & 152 \\
\hline & After & $\ldots$ & 139 & 253 & 270 & - & 178 \\
\hline \multirow[t]{2}{*}{5} & Before & $\ldots$ & 143 & 183 & 148 & - & 111 \\
\hline & After & $\ldots$ & 103 & 181 & 155 & - & 105 \\
\hline \multirow[t]{2}{*}{6} & Before & $\ldots$ & 129 & 327 & 363 & 392 & - \\
\hline & After & $\ldots$ & 91 & 220 & 234 & 241 & 156 \\
\hline \multirow[t]{2}{*}{7} & Before & $\ldots$ & 124 & 160 & 142 & - & 125 \\
\hline & After & $\ldots$ & 70 & 136 & 133 & - & 107 \\
\hline \multirow[t]{2}{*}{8} & Before & $\ldots$ & 140 & 488 & 442 & - & 184 \\
\hline & After & $\ldots$ & 102 & 243 & 189 & - & 93 \\
\hline \multirow[t]{2}{*}{9} & Before & $\ldots$ & 139 & 218 & 156 & - & 114 \\
\hline & After & $\ldots$ & 115 & 175 & 146 & - & 114 \\
\hline \multirow[t]{2}{*}{10} & Before & $\ldots$ & 110 & 144 & 164 & 142 & - \\
\hline & After & $\ldots$ & 125 & 148 & 172 & 166 & 181 \\
\hline Control 1 & $\ldots$ & $\ldots$ & 120 & 149 & 121 & 98 & - \\
\hline Control 2 & $\ldots$ & $\ldots$ & 95 & 196 & 168 & 114 & - \\
\hline Control 3 & $\ldots$ & $\ldots$ & 99 & 165 & 185 & 156 & - \\
\hline Control 4 & $\cdots$ & $\ldots$ & 104 & 152 & 218 & 214 & - \\
\hline Control 5 & $\cdots$ & $\ldots$ & 98 & 155 & 219 & 141 & - \\
\hline
\end{tabular}


maximum dose in grammes per kilogramme of body weight had been used. The particularly high curves obtained in some of the cases gave rise to some doubt whether this dictum was applicable in children. For this reason five control curves were made on normal children about the same age, and as these were found to be within normal limits, it is suggested that these high values were due in most cases to sepsis, or where no obvious focus of pyogenic infection could be discovered, to the original tuberculous disease itself.

The resting sugar was taken at least three hours after a meal and the patients were then given 80 grm. of powdered glucose in water. Blood was taken from a vein at half, one and two-hourly intervals. The proteins were precipitated immediately in almost all cases and the blood sugar estimated by the colorimetric method (Folin and $\mathrm{Wu}$ ).

\section{Conclusions.}

(1) It would appear that in patients suffering from tuberculous lesions the carbohydrate tolerance is increased following exposures to ultra-violet radiation.

(2) As has been stated no conclusion regarding any association between carbohydrate metabolism, ultra-violet radiation, and the suprarenal capsules can be assumed, because so many other aspects must be taken into consideration. Such an association may, however, be suspected.

\section{EXPERIMENTAL DATA \& CONCLUSIONS.}

\section{Experimental Data.}

The following charts represent the numerical readings of the tests involved ; but it must be emphasized that the conclusions are based not only on these tests, but also in large part on the clinical picture presented by each case, $\mathrm{X}$-Ray reports, the formation and breaking down of abscesses, temperature range, etc.

In certain cases, also, ultra-violet therapy, and/or endocrine therapy, were stopped for occasional periods and the effect on the patient noted. These and other observations have of necessity been omitted, but have been taken into consideration in forming conclusions. It is to be regretted that a time limit had to be fixed for the duration of the tests, and in consequence certain conclusions are immature.

The following scheme has been drawn up with a view to simplifying the results :-

A. Central Lesions.

C.1. Early.

C.2. Moderately advanced but without sinus formation.

C.3, Advanced with sinuses. 


\section{B. Peripheral Lesions.}

The term 'central lesion' is used to imply a lesion in one of the major joints, spine, hip or knee.

Under the heading 'peripheral lesion' are included lesions of the smaller joints, ankle, elbow, and wrist, adenitis, skin lesions and peritonitis.

\section{Conclusions.}

\section{General Conclusions.}

It will be seen from the cases recorded that following the first month's treatment with ultra-violet radiation, many cases show a fall in the sedimentation rate reading, followed by a rise in the succeding month. This initial fall has been so frequently noted that for descriptive purposes it has been called ' the negative phase.' It will be further noticed that in other cases which are secondarily infected, or in which an abscess is developing, the succeeding rise is at least delayed. It is thought that the significance of this fall is an increased reaction in the focus of disease.

It is also considered that the severity of this reaction is dependent upon three factors :-(1) The severity and site of the lesion ; $(2)$ The degree of mixed infection, if present ; and (3) The dosage of ultra-violet radiation.

These three factors together with the reaction to light of the individual may, it is thought, provide a basis upon which this adjuvant method of treatment should be prescribed.

Of these three factors there is only one, namely, radiation, which can be varied at will. It is felt that the method of treatment in vogue at present is too empirical, and that this source of natural energy should be used in prescribed doses according to the reaction which might be expected to occur. It is considered that in general diseases a mild response, or possibly a single 'shock' dose, is more desirable than a continued severe, or moderately severe reaction.

Possibly the nearer natural sunshine is simulated for general diseases, and this can be best done by using carbon-arc lamps with mild carbons, the better the results will be. It is felt that adequate control of dosage could be obtained by observations of temperature, sedimentation rate, and skiagrams.

Subject to consideration of the above-mentioned controlling factors, it is thought that in ultra-violet radiation is to be found an adjuvant method of treatment of considerable value and possibility in cases of tuberculosis in children,

\section{Special Conclusions.}

A. Central Lesions. (1) Early. It is to be regretted that the number of cases under this heading is small, but from these and the suspected action of ultra-violet radiation it is thought that in this group radiation is of considerable value. It is considered that small doses are probably best, possibly after three months immobilization, when the resistance of the body is expected to be on the up grade. 
(2) Moderately advanced, but without sinus formation. The clinical impression gained from cases in this group is that there has been a better response to treatment in cases receiving radiation than in corresponding cases in hospital. The sedimentation rates in this section, it is thought, have not been taken over a sufficiently long period in some cases, for where abscesses are present it is to be expected that until they heal completely the sedimentation rate will remain low.

In this group again it is considered there is scope for ultra-violet radiation, but only where the dose is graduated according to the principles laid down in the introduction to this section. Here again the influence of possible secondary infection, although the skin is unbroken, is felt to be of moment.

(3) Advanced, with sinuses. With but one exception the results in this group have been disappointing. In the majority that the activity of the disease has been increased to a considerable extent after irradiation is shown by lower sedimentation-rate readings, lower blood pressure, wasting, increased discharge from sinuses, and in three cases by death. It is considered that ultra-violet radiation accelerated this result.

It would appear that in the presence of a large focus of pyogenic infection, radiation either lowers the bodily resistance or activates the bacteria to a much greater extent than the resistance of the body can withstand.

Although the results obtained in this group have been poor it must be remembered that the dosage in all cases was empirical and that no change was made from the routine. It is possible that if this dose could be controlled and this type of case receive a much shorter exposure, by coaxing the resistance of the body some benefit might be obtained.

B. Peripheral Lesions. The treatment of peripheral lesions by ultraviolet radiation is the most favourable field for this mode of therapy.

Disease of the ankle and elbow joints, with or without sinuses do well, and also tuberculous skin lesions. It is of interest to note that the sinuses of peripheral lesions heal more rapidly with radiation than without.

Cases of tuberculous peritonitis, diagnosed clinically or with physical signs, improve immensely, except those which are advanced. There is also a feeling that the stay in hospital of these cases is shortened.

Glands of neck. It was noticed that following a few exposures to radiation in a number of cases of cervical adenitis the glands broke down, with extrusion of their contents through the skin, but that in others, after first becoming further enlarged and rather tender, they retracted and became extremely hard. It is thought that the condition of the glands as regards softening at the commencement of treatment, and particularly the influence of local sepsis, teeth, tonsils, etc., play some part in the reaction of the glands to radiation. Great care is required in treating cases of cervical adenitis, and the dose must be carefully regulated. Where there is a sinus present good results were almost always obtained.

In conclusion I would like to thank Dr. A. Dingwall Fordyce for his invaluable encouragement and advice. 


\section{REFERENCES.}

1. Fohrous, R., Hygiea, Stockholm, 1918, LXXX, 369.

2. Westergren, A., Brit. J. Tuberc., Lond., 1921, XV, 72.

3. Heaf, F. R. G., Tubercle., Lond., 1926-27, VIII, 97.

4. Cummins, S. L. \& Acland, C. M., Ibid., 1927-28, IX, 1.

5. Bannerman, R. G., Brit. J. Tuberc., Lond., 1927, XXI, 207.

7. Beaumont, G. E. \& Dodds, E. C., Recent Advances in Medicine, Lond., 1926, 3rd Ed., 339.

8. Mayer, E., Clin. Applic. of Sunlight, etc., Lond., 1926, 157.

9. Loc. cit., 158.

11. Schafer, E. S., Endocrine Organs, 1924, 2nd Ed., Pt. 1, 93.

12. Ferguson, J. B., Quartz Mercury Vapour Lamp, Lond., 1926, 28.

13. Lancet, Lond., 1927; II, 649.

14. Brit. Med. J., Lond., 1927, II, 258.

15. Ferguson, J. B., Quartz Mercury Vapour Lamp, Lond., 1926, 13.

16. Loc. cit., 29.

17. Mathews, A. P., Physiological Chemistry, Baltimore, 1921, 3rd Ed., 675

18. Vincent, S., Int. Secretion \& Ductless Glands, Lond., 1924, 3rd Ed., 161.

19. Grant, J. H. B., \& Gates, F. L., Proc. Soc. Exp. Biol. \& Med., N.Y., 1925, XXII, 315.

20. Ashby, H. T., Quart. J. Med., Oxf., 1925-26, XIX, 375.

21. Vincent, S., Int. Secretion \& Ductless Glands, Lond., 1924, 3rd Ed., 451.

22. Beaumont, G. E., \& Dodds, E. C., Recent Advances in Medicine, Lond., 1926, 3rd Ed., 213.

23. Cannon, W. B., Amer. J. Physiol., Boston, 1911. 